\title{
Generalized conditional entropy in bipartite quantum systems
}

\author{
N. Gigena and R. Rossignoli \\ Departamento de Física-IFLP, Universidad Nacional de La Plata, C.C. 6\%, La Plata (1900), Argentina
}

\begin{abstract}
We analyze, for a general concave entropic form, the associated conditional entropy of a quantum system $A+B$, obtained as a result of a local measurement on one of the systems $(B)$. This quantity is a measure of the average mixedness of $A$ after such measurement, and its minimum over all local measurements is shown to be the associated entanglement of formation between $A$ and a purifying third system $C$. In the case of the von Neumann entropy, this minimum determines also the quantum discord. For classically correlated states and mixtures of a pure state with the maximally mixed state, we show that the minimizing measurement can be determined analytically and is universal, i.e., the same for all concave forms. While these properties no longer hold for general states, we also show that in the special case of the linear entropy, an explicit expression for the associated conditional entropy can be obtained, whose minimum among projective measurements in a general qudit-qubit state can be determined analytically, in terms of the largest eigenvalue of a simple $3 \times 3$ correlation matrix. Such minimum determines the maximum conditional purity of $A$, and the associated minimizing measurement is shown to be also universal in the vicinity of maximal mixedness. Results for $X$ states, including typical reduced states of spin pairs in $X Y$ chains at weak and strong transverse fields, are also provided and indicate that the measurements minimizing the von Neumann and linear conditional entropies are typically coincident in these states, being determined essentially by the main correlation. They can differ, however, substantially from that minimizing the geometric discord.
\end{abstract}

PACS numbers: 03.67.-a, 03.65.Ud, 03.65.Ta

\section{INTRODUCTION}

There is presently a great interest in the investigation of quantum correlations in mixed states of composite quantum systems. While for pure states such correlations can be identified with entanglement, the situation in mixed states is more complex, as separable (non entangled) mixed states, defined as convex mixtures of product states [1] (i.e., states which can be generated by local operations and classical communication), can still exhibit signatures of quantum-like correlations, manifested for instance in a non-zero quantum discord 2, 4]. Interest on this quantity has been enhanced by the existence of mixed state based quantum algorithms [5] able to achieve an exponential speed-up over the corresponding classical algorithm with vanishing entanglement [6] yet finite discord [7. Various operational interpretations and implications of states with non-zero discord have been recently provided 811 .

The quantum discord for a bipartite system $A+B$ can be written [2] as the minimum difference between two distinct quantum extensions of the classical Shannon based conditional entropy $S(A \mid B)$ [12], one involving a local measurement $M_{B}$ on one of the systems $(B)$, over which the minimization is to be performed, and the other the direct quantum version of the classically equivalent expression $S(A, B)-S(B)$ (which becomes negative in pure entangled states). While other measures of quantum correlations with similar properties (like reducing to an entanglement measure for pure states and vanishing just for classically correlated states) have been introduced [4, 8, 9, 11, 13, 22], the quantum discord has the special feature, due to its definition through a conditional entropy, of being directly related with the entanglement of formation between the unmeasured system and a third system which purifies the whole system 23 26. Accordingly, the measurement minimizing the quantum discord can differ substantially from those minimizing other measures such as the geometric discord [8, 16], which can be much more easily determined. The complex minimization involved in the quantum discord has in fact limited its evaluation to simple systems or special states and measurements $\mathbf{7}, 8,2734$.

The aim of this work is first to extend the concept of measurement dependent conditional entropy to a general entropic form (or uncertainty measure) $S_{f}$ depending on an arbitrary concave function $f$ [12, 35]. The ensuing quantity $S_{f}\left(A \mid B_{M_{B}}\right)$ provides a measure of the average conditional mixedness of $A$ after a measurement at $B$, and allows to define an associated generalized "information gain" or uncertainty reduction $I_{f}\left(A \mid B_{M_{B}}\right)=$ $S_{f}(A)-S_{f}\left(A \mid B_{M_{B}}\right)$, which is non-negative for any concave $f$ and reduces to the associated entanglement entropy $S_{f}(A)$ in the case of pure states. Such extension differs then from other treatments 36 38 dealing with the generalization of the measurement independent von Neumann conditional entropy $S(A, B)-S(B)$. The minimum of the present $S_{f}\left(A \mid B_{M_{B}}\right)$ among all local measurements coincides with the associated entanglement of formation (convex roof extension of the $S_{f}$ entanglement entropy) between $A$ and a purifying third system $C$, as will be shown.

Such general formulation allows, first, to recognize some universal features of the measurement dependent conditional entropy which do not depend on the choice of entropic function $f$ and rely just on concavity. It also 
opens the way to use simple entropic forms like the linear entropy $S_{2}(\rho)=1-\operatorname{Tr} \rho^{2}$, trivially related with the purity $P(\rho)=\operatorname{Tr} \rho^{2}$ and lower bound to the von Neumann entropy, which can be more easily evaluated (it does not require the eigenvalues of $\rho$ ) and can therefore help to determine and understand the minimizing measurement of the von Neumann conditional entropy and hence the quantum discord. Moreover, we will show that this entropy determines the behavior of all entropies in the vicinity of the maximally mixed state. The purity, and hence $S_{2}(\rho)$, is also more easily accessible from the experimental side, since it can be determined efficiently without requiring a full state tomography [39].

We first derive in sec. 2 the fundamental properties of $S_{f}\left(A \mid B_{M_{B}}\right)$, including its minimum in general classically correlated states and mixtures of a pure state with the maximally mixed state, where the minimizing measurement is shown to be universal, i.e., the same for any entropic form. The formalism is then applied in sec. 3 to derive a closed expression for the conditional $S_{2}$ entropy and discuss its fundamental properties, including its minimum over projective measurements for a general $A+$ qubit system, which is shown to be determined by the largest eigenvalue of a simple $3 \times 3$ contracted correlation matrix. This permits to easily recognize the minimizing measurement and understand its behavior. Applications to general parity preserving two-qubit states ( $X$ states), including mixtures of aligned states and weakly correlated states, relevant for the description of pair states in interacting $X Y$ spin chains at weak and strong transverse fields, are presented in sec. 4. These examples indicate a similar behavior (and coincidence of the minimizing measurement) of the $S_{2}$ and von Neumann conditional entropies for these states, even well beyond the vicinity of maximal mixedness. Conclusions are finally given in sec. 5 .

\section{FORMALISM}

\section{A. Generalized conditional entropy after a local measurement}

We consider a bipartite quantum system $A+B$ in an initial state $\rho \equiv \rho_{A B}$, with reduced states $\rho_{A}=\operatorname{Tr}_{B} \rho$, $\rho_{B}=\operatorname{Tr}_{A} \rho$. We assume a general positive operator valued local measurement 40] $M_{B}$ on system $B$ is performed, defined by a set of operators $M_{j}=I_{A} \otimes M_{j}^{B}$, $j=1, \ldots, j_{m}$, such that the state after outcome $j$ is proportional to $M_{j} \rho M_{j}^{\dagger}$. The positive semidefinite operators

$$
\Pi_{j}=M_{j}^{\dagger} M_{j}=I_{A} \otimes \Pi_{j}^{B},
$$

should then satisfy $\sum_{j} \Pi_{j}=I \equiv I_{A} \otimes I_{B}$.

The reduced state of $A$ after outcome $j$ depends just on $\Pi_{j}$ and is given by

$$
\rho_{A / \Pi_{j}}=p_{j}^{-1} \operatorname{Tr}_{B} \rho \Pi_{j}, \quad p_{j}=\operatorname{Tr} \rho \Pi_{j},
$$

where $p_{j}>0$ is the probability of such outcome. In order to quantify the average uncertainty or mixedness of the state of $A$ after such measurement, we will consider here the generalized conditional entropy

$$
S_{f}\left(A \mid B_{\left\{\Pi_{j}\right\}}\right)=\sum_{j} p_{j} S_{f}\left(\rho_{A / \Pi_{j}}\right),
$$

where

$$
S_{f}(\rho)=\operatorname{Tr} f(\rho)
$$

represents a generalized entropic form or uncertainty measure [12, 35] (see A). Here $f:[0,1] \rightarrow \Re$ is a smooth strictly concave function satisfying $f(0)=f(1)=0$. For $f(\rho)=-\rho \log _{a} \rho$ (we use here $a=2$ or $e$ ), $S_{f}(\rho)$ becomes the von Neumann entropy $S(\rho)=-\operatorname{Tr} \rho \log _{a} \rho$, and Eq. (3) the measurement dependent von Neumann conditional entropy, introduced in 2] for the definition of the quantum discord.

The concavity of these forms, i.e.,

$$
S_{f}\left(\sum_{\alpha} q_{\alpha} \rho_{\alpha}\right) \geq \sum_{\alpha} q_{\alpha} S_{f}\left(\rho_{\alpha}\right),
$$

if $\left\{q_{\alpha}\right\}$ is a probability distribution $\left(q_{\alpha} \geq 0, \sum_{\alpha} q_{\alpha}=1\right)$ and all $\rho_{\alpha}$ 's are quantum states, directly follows from the concavity of $f$, and implies fundamental properties of the generalized conditional entropy (3). First, since $\rho_{A}=\sum_{j} p_{j} \rho_{A / \Pi_{j}}$, Eq. (5) implies $S_{f}(A) \equiv S_{f}\left(\rho_{A}\right) \geq$ $\sum_{j} p_{j} S_{f}\left(\rho_{A / \Pi_{j}}\right)$, i.e.,

$$
S_{f}(A) \geq S_{f}\left(A \mid B_{\left\{\Pi_{j}\right\}}\right)
$$

indicating that the average conditional mixedness of $A$ after a measurement at $B$, will not exceed the original mixedness, for any choice of $S_{f}$. Moreover, if $f$ is strictly concave, equality in (5) holds iff all $\rho_{\alpha}$ 's with $q_{\alpha}>0$ are identical. Hence, equality in (6) for all $M_{B}$ holds just if $\rho=\rho_{A} \otimes \rho_{B}$, since only in this case $\rho_{A / \Pi_{j}}=\rho_{A} \forall \Pi_{j}$. The quantity

$$
I_{f}\left(A \mid B_{\left\{\Pi_{j}\right\}}\right)=S_{f}(A)-S_{f}\left(A \mid B_{\left\{\Pi_{j}\right\}}\right),
$$

is then non-negative for any $S_{f}$, vanishing for all $M_{B}$ just for product states. It represents the average reduction in the quantum uncertainty of $A$ (or generalized information gain about $A$ ) as measured by $S_{f}$, after a measurement at $B$.

Eq. (5) also implies concavity of the conditional entropy: If $\rho=\sum_{\alpha} q_{\alpha} \rho^{\alpha}$, then $\rho_{A / \Pi_{j}}=$ $\sum_{\alpha} p_{j}^{-1} q_{\alpha} p_{j}^{\alpha} \rho_{A / \Pi_{j}}^{\alpha}$, with $p_{j}^{\alpha}=\operatorname{Tr} \rho^{\alpha} \Pi_{j}, p_{j}=\sum_{\alpha} q_{\alpha} p_{j}^{\alpha}$. Hence, $S_{f}\left(\rho_{A / \Pi_{j}}\right) \geq \sum_{\alpha} p_{j}^{-1} q_{\alpha} p_{j}^{\alpha} S_{f}\left(\rho_{A / \Pi_{j}}^{\alpha}\right)$, entailing

$$
S_{f}\left(A \mid B_{\left\{\Pi_{j}\right\}}\right) \geq \sum_{\alpha} q_{\alpha} S_{f}\left(A^{\alpha} \mid B_{\left\{\Pi_{j}\right\}}^{\alpha}\right),
$$

where $S_{f}\left(A^{\alpha} \mid B_{\left\{\Pi_{j}\right\}}^{\alpha}\right)=\sum_{j} p_{j}^{\alpha} S_{f}\left(\rho_{A / \Pi_{j}}^{\alpha}\right)$ : Average uncertainty about $A$ after state mixing cannot be smaller 
than the average of the original average uncertainties. In addition, if

$$
\Pi_{j}=\sum_{k} r_{j}^{k} \tilde{\Pi}_{k}, \quad r_{j}^{k} \geq 0
$$

where $\tilde{\Pi}_{k}=I_{A} \otimes \tilde{\Pi}_{k}^{B}$, with $\sum_{k} \tilde{\Pi}_{k}=I$, are positive operators representing a more detailed local measurement (i.e., all $\tilde{\Pi}_{k}^{B}$ of rank 1) and $\sum_{j} r_{j}^{k}=1$, then $\rho_{A / \Pi_{j}}=$ $\sum_{k} p_{j}^{-1} r_{j}^{k} q_{k} \rho_{A / \tilde{\Pi}_{k}}$, with $q_{k}=\operatorname{Tr} \rho \tilde{\Pi}_{k}, \sum_{k} r_{j}^{k} q_{k}=p_{j}$. Hence, $S_{f}\left(\rho_{A / \Pi_{j}}\right) \geq \sum_{k} p_{j}^{-1} r_{j}^{k} q_{k} S_{f}\left(\rho_{A / \tilde{\Pi}_{k}}\right)$ and

$$
S_{f}\left(A \mid B_{\left\{\Pi_{j}\right\}}\right) \geq \sum_{k} q_{k} S_{f}\left(\rho_{A / \tilde{\Pi}_{k}}\right)=S_{f}\left(A \mid B_{\left\{\tilde{\Pi}_{k}\right\}}\right),
$$

i.e., the generalized conditional entropy will not increase (and will in general decrease) if a more detailed local measurement is performed. In fact, $S_{f}(A)$ can be considered as the conditional entropy $S_{f}\left(A \mid B_{I}\right)$ of $A$ after a trivial measurement of the identity $I_{B}$ in $B$, so that Eq. (6) is a particular case of (10).

Minimum uncertainty about the state of $A$ will then be obtained for measurements based on rank one operators

$$
\Pi_{j}^{B}=r_{j}\left|j_{B}\right\rangle\left\langle j_{B}\right|, \quad r_{j}>0,
$$

where $\left|j_{B}\right\rangle$ are normalized states such that $\sum_{j} \Pi_{j}^{B}=I_{B}$. Standard complete projective measurements (von Neumann measurements) correspond to $r_{j}=1$ and $\left\{\left|j_{B}\right\rangle\right\}$ an orthonormal basis $\left(\Pi_{j} \Pi_{j^{\prime}}=\delta_{j j^{\prime}} \Pi_{j}\right)$. In particular, for pure states $\rho^{2}=\rho$, i.e.,

$$
\rho=|\Psi\rangle\langle\Psi|, \quad| \Psi\rangle=\sum_{k} \sqrt{q_{k}}\left|\tilde{k}_{A} \tilde{k}_{B}\right\rangle,
$$

where the last expression denotes the Schmidt decomposition [40] ( $\left\{\left|k_{A}\right\rangle\right\},\left\{\left|k_{B}\right\rangle\right\}$ orthonormal sets), $\rho_{A / \Pi_{j}}$ is pure $\forall j$ with $p_{j}>0$, for any local measurement based on the operators (11):

$\rho_{A / \Pi_{j}}=\left|j_{A}\right\rangle\left\langle j_{A}|, \quad| j_{A}\right\rangle=\left(r_{j} / p_{j}\right)^{1 / 2} \sum_{k} \sqrt{q_{k}}\left\langle j_{B} \mid \tilde{k}_{B}\right\rangle\left|\tilde{k}_{A}\right\rangle$

where $p_{j}=r_{j} \sum_{k} q_{k}\left|\left\langle j_{B} \mid \tilde{k}_{B}\right\rangle\right|^{2}$. Hence, in the pure case $S_{f}\left(A \mid B_{\left\{\Pi_{j}\right\}}\right)=0$, and Eq. (77) becomes the generalized entanglement entropy [17]:

$$
I_{f}\left(A \mid B_{\left\{\Pi_{j}\right\}}\right)=S_{f}(A)=S_{f}(B)=\sum_{k} f\left(q_{k}\right) .
$$

\section{B. Minimum conditional entropy and generalized entanglement of formation}

Let us now consider the minimum of Eq. (3) among all local measurements $M_{B}$ for a general state $\rho$,

$$
S_{f}(A \mid B) \equiv \operatorname{Min}_{\left\{\Pi_{j}\right\}} S_{f}\left(A \mid B_{\left\{\Pi_{j}\right\}}\right) .
$$

From Eq. (10) it follows that just rank one operators of the form (11) need to be considered in the minimization. Eq. 15 leads to the maximum generalized information gain (i.e., maximum uncertainty reduction)

$$
I_{f}(A \mid B)=\operatorname{Max}_{\left\{\Pi_{j}\right\}} I_{f}\left(A \mid B_{\left\{\Pi_{j}\right\}}\right)=S_{f}(A)-S_{f}(A \mid B) .
$$

If the system $A+B$ is purified [40] by a adding a third system $C$, Eq. (15) has the important meaning of being the associated entanglement of formation $E_{f}(A, C)$ [17] between $A$ and $C$ in the reduced state $\rho_{A C}$ 223]:

$$
S_{f}(A \mid B)=E_{f}(A, C)=\underset{\sum_{j} p_{j} \rho_{A C}^{j}=\rho_{A C}}{\operatorname{Min}} \sum_{j} p_{j} S_{f}\left(\rho_{A}^{j}\right),
$$

where the minimization is over all representations of $\rho_{A C}$ as convex combination $\left(p_{j}>0\right)$ of pure states $\rho_{A C}^{j}=$ $\left|j_{A C}\right\rangle\left\langle j_{A C}\right|$, and $S_{f}\left(\rho_{A}^{j}\right)=S_{f}\left(\rho_{C}^{j}\right)$ is the $S_{f}$ entanglement entropy between $A$ and $C$ in $\left|j_{A C}\right\rangle\left(\rho_{A}^{j}=\operatorname{Tr}_{C} \rho_{A C}^{j}\right)$. Eq. [17] is the convex roof extension [4] of the pure state entanglement entropy (14) and is an entanglement monotone 42]. The identity (17) was derived for the von Neumann entropy (see 23] and 24 26]), where $E_{f}(A, C)$ becomes the standard entanglement of formation $E(A, C)$ [43], but the arguments remain valid in the present general case (see B).

Eq. (17) entails that the Eq. (16) can be also expressed as

$$
I_{f}(A \mid B)=E_{f}(A, B C)-E_{f}(A, C),
$$

where $E_{f}(A, B C)=S_{f}\left(\rho_{A}\right)=S_{f}\left(\rho_{B C}\right)$ is the entanglement entropy between $A$ and $B C$ in the purified state.

The quantum discord 2] 4, \&] $D(A \mid B)$, as obtained by a measurement in $B$, is directly related to the present von Neumann conditional entropy $S\left(A \mid B_{\left\{\Pi_{j}\right\}}\right)$ through

$$
D(A \mid B)=\operatorname{Min}_{\left\{\Pi_{j}\right\}} S\left(A \mid B_{\left\{\Pi_{j}\right\}}\right)-[S(A, B)-S(B)],
$$

where the last bracket is the standard (measurement independent) quantum extension of the von Neumann conditional entropy (which can be negative in entangled states). It can be also expressed as the difference between the standard mutual information $S(A)+S(B)-$ $S(A, B)$ and the maximum von Neumann information gain $I(A \mid B)=S(A)-\operatorname{Min}_{\left\{\Pi_{j}\right\}} S\left(A \mid B_{\left\{\Pi_{j}\right\}}\right)$. A generalization of the quantum discord based on the Renyi entropy of order 2 was considered in 22] for gaussian states, whereas extensions based on the Tsallis entropy [4] were discussed in 45].

\section{Classically correlated states}

There are important classes of mixed states where the local measurement minimizing $S_{f}\left(A \mid B_{\left\{\Pi_{j}\right\}}\right)$ is universal, i.e., the same for all entropies $S_{f}$, and can be generally 
determined. One is that of classically correlated states with respect to $B$ 2 4 ,

$$
\rho=\sum_{k} q_{k} \rho_{A / k} \otimes \tilde{\Pi}_{k}^{B},
$$

where $q_{k} \geq 0$ and $\left\{\tilde{\Pi}_{k}^{B}=\left|\tilde{k}_{B}\right\rangle\left\langle\tilde{k}_{B}\right|\right\}$ is a complete set of orthogonal rank one local projectors, such that after a local measurement in this basis, $\rho_{A / \tilde{\Pi}_{k}}=\rho_{A / k}$ (and $\sum_{k} \tilde{\Pi}_{k} \rho \tilde{\Pi}_{k}=\rho$ if $\tilde{\Pi}_{k}=I_{A} \otimes \tilde{\Pi}_{k}^{B}$, implying that the states (20) remain unchanged after an unread local measurement in this basis). It is easy to prove that the lowest conditional entropy (15) is obtained for such measurement, for any $S_{f}$ :

$$
S_{f}(A \mid B)=S_{f}\left(A \mid B_{\left\{\tilde{\Pi}_{k}\right\}}\right)=\sum_{k} q_{k} S_{f}\left(\rho_{A / k}\right) .
$$

Proof: For any $M_{B}$ based on the operators (11), we have

$$
\rho_{A / \Pi_{j}}=\sum_{k} r_{j} p_{j}^{-1} q_{k}\left|\left\langle j_{B} \mid \tilde{k}_{B}\right\rangle\right|^{2} \rho_{A / k},
$$

with $p_{j}=r_{j} \sum_{k} q_{k}\left|\left\langle j_{B} \mid \tilde{k}_{B}\right\rangle\right|^{2}$. Concavity plus completeness $\left(\sum_{j} r_{j}\left|\left\langle j_{B} \mid \tilde{k}_{B}\right\rangle\right|^{2}=1\right)$ imply

$$
\begin{aligned}
S_{f}\left(A \mid B_{\left\{\Pi_{j}\right\}}\right) & \geq \sum_{k, j} r_{j} q_{k}\left|\left\langle j_{B} \mid \tilde{k}_{B}\right\rangle\right|^{2} S_{f}\left(\rho_{A / k}\right) \\
& =\sum_{k} q_{k} S_{f}\left(\rho_{A / k}\right),
\end{aligned}
$$

with the inequality saturated for a measurement in the pointer basis $\left\{\left|\tilde{k}_{B}\right\rangle\right\}$, formed by the eigenstates of $\rho_{B}=$ $\sum_{k} q_{k} \tilde{\Pi}_{k}^{B}$. The maximum $I_{f}$ is then

$$
I_{f}(A \mid B)=S_{f}\left(\sum_{k} q_{k} \rho_{A / k}\right)-\sum_{k} q_{k} S_{f}\left(\rho_{A / k}\right) .
$$

\section{Pure state plus maximally mixed state}

A second case is that of the mixture of a general pure state (12) with the maximally mixed state $I / d$,

$$
\rho=w|\Psi\rangle\left\langle\Psi\left|+(1-w) I_{d} / d, \quad\right| \Psi\right\rangle=\sum_{k} \sqrt{q_{k}}\left|\tilde{k}_{A} \tilde{k}_{B}\right\rangle,
$$

where $w \in[0,1]$ and $d=d_{A} d_{B}$ is the Hilbert-space dimension of $A+B$. The minimum for any $S_{f}$ is provided again by a measurement in the basis $\left\{\left|\tilde{k}_{B}\right\rangle\right\}$ of eigenstates of $\rho_{B}$ :

$$
\begin{aligned}
S_{f}(A \mid B) & =S\left(A \mid B_{\left\{\tilde{\Pi}_{k}\right\}}\right)=\sum_{k} q_{k}^{w} S_{f}\left(\rho_{A / \tilde{\Pi}_{k}}\right) \\
& =\sum_{k} q_{k}^{w}\left[f\left(\frac{w q_{k}+(1-w) / d}{q_{k}^{w}}\right)+\left(d_{A}-1\right) f\left(\frac{1-w}{d q_{k}^{w}}\right)\right],
\end{aligned}
$$

where $q_{k}^{w}=w q_{k}+\frac{1-w}{d_{B}}$ is the probability of outcome $k$ at $B$ and $\rho_{A / \tilde{\Pi}_{k}}=\left[w q_{k}\left|\tilde{k}_{A}\right\rangle\left\langle\tilde{k}_{A}\right|+(1-w) I_{A} / d\right] / q_{k}^{w}$ the state of $A$ after such outcome.

Proof: For any measurement based on the operators (11) we obtain, using (12)-(13),

$$
\begin{aligned}
\rho_{A / \Pi_{j}} & =\frac{w p_{j}\left|j_{A}\right\rangle\left\langle j_{A}\right|+r_{j}(1-w) I_{A} / d}{p_{j}^{w}} \\
& =\sum_{k} \frac{r_{j} q_{k}^{w}}{p_{j}^{w}}\left|\left\langle j_{B} \mid \tilde{k}_{B}\right\rangle^{2}\right| U_{k}^{j} \rho_{A / \tilde{\Pi}_{k}} U_{k}^{j^{\dagger}},
\end{aligned}
$$

where $p_{j}=r_{j} \sum_{k} q_{k}\left|\left\langle j_{B} \mid \tilde{k}_{B}\right\rangle\right|^{2}$ and $p_{j}^{w}=w p_{j}+r_{j} \frac{1-w}{d_{B}}$ are respectively the probabilities of outcome $j$ in $|\Psi\rangle$ and $\rho$, and $U_{k}^{j}$ are unitaries satisfying $U_{k}^{j}\left|\tilde{k}_{A}\right\rangle=\left|j_{A}\right\rangle$. Hence, concavity, invariance of $S_{f}$ under unitary transformations and completeness imply again

$$
S_{f}\left(A \mid B_{\left\{\Pi_{j}\right\}}\right) \geq \sum_{k} q_{k}^{w} S_{f}\left(\rho_{A / \tilde{\Pi}_{k}}\right)=S_{f}\left(A \mid B_{\left\{\tilde{\Pi}_{k}\right\}}\right)
$$

Equality in 28) for any $M_{B}$ of the form (11) holds for i) $w=0$ ( $\rho$ maximally mixed $),$ ii $) w=1$ ( $\rho$ pure $)$ and iii) $|\Psi\rangle$ maximally entangled $\left(q_{k}=1 / d_{B} \forall k\right.$, assuming $d_{A} \geq$ $\left.d_{B}\right)$, where $p_{j}=r_{j} / d_{B} \forall j$ and all $\rho_{A / \Pi_{j}}=w\left|j_{A}\right\rangle\left\langle j_{A}\right|+$ $\frac{1-w}{d_{A}} I_{A}$ have the same spectrum.

It can be easily checked that Eq. 26] is a concave function of both $w$ and the probability distribution $\boldsymbol{q}=$ $\left\{q_{k}\right\}$. Since $S_{f}(A \mid B)$ reaches its maximum $S_{f}\left(I_{A} / d_{A}\right)=$ $d_{A} f\left(1 / d_{A}\right)$ for $w=0$, concavity entails that Eq. (26) is a decreasing function of $w$ for $w \in[0,1] \forall S_{f}$ : Decreasing mixedness decreases the uncertainty about $A$. Concavity also leads to the immediate lower bound $S_{f}(A \mid B) \geq(1-$ $w) d_{A} f\left(1 / d_{A}\right)$.

Besides, for states $|\Psi\rangle,\left|\Psi^{\prime}\right\rangle$ characterized by distributions $\boldsymbol{q}$ and $\boldsymbol{q}^{\prime}$ in the Schmidt decomposition, we have $S_{f}(\boldsymbol{q}) \geq S_{f}\left(\boldsymbol{q}^{\prime}\right) \forall S_{f}$ iff $\boldsymbol{q} \prec \boldsymbol{q}^{\prime}$ (i.e., $\boldsymbol{q}$ majorized by $\boldsymbol{q}^{\prime}$, see . Such condition ensures then that $|\Psi\rangle$ is more entangled than $\left|\Psi^{\prime}\right\rangle$ for any $S_{f}$, and is the same condition which warrants that $\left|\Psi^{\prime}\right\rangle$ can be obtained from $|\Psi\rangle$ by LOCC 40, 46]. In such a case, concavity of $S_{f}(A \mid B)$ with respect to $\boldsymbol{q}$ entails that at fixed $w \in(0,1)$, $S_{f}(A \mid B)_{|\Psi\rangle} \geq S_{f}(A \mid B)_{\left|\Psi^{\prime}\right\rangle}$ for any $S_{f}$, i.e., greater entanglement for any $S_{f}$ entails a larger conditional entropy $S_{f}(A \mid B) \forall S_{f}$ in the mixture (25), in contrast with the pure case $w=1$ (where $S_{f}(A \mid B)=0$ for any pure state $|\Psi\rangle)$.

\section{THE QUADRATIC CASE: CONDITIONAL PURITY AFTER LOCAL MEASUREMENT}

\section{A. General properties}

We now consider in detail the simplest choice of concave $f$, i.e., a quadratic function $f(\rho)=\alpha\left(\rho-\rho^{2}\right), \alpha>0$. For $\alpha=1$ this leads to $S_{f}(\rho)=S_{2}(\rho)$, with

$$
S_{2}(\rho)=1-\operatorname{Tr} \rho^{2},
$$


the so called linear entropy, since it corresponds to the linear approximation $-\ln \rho \approx I-\rho$ in $S(\rho)(\ln p=p-$ $1+O(p-1)^{2}$ for $\left.p \rightarrow 1\right)$. It is the $q=2$ case of the Tsallis entropy $S_{q}(\rho)$ 44 (see $\mathrm{A}$ ) and provides a lower bound to the von Neumann entropy for $a=e$ (and hence $a<e$ ), since $p(1-p) \leq-p \ln p \forall p \in[0,1]$.

Eq. (29) is trivially related with the purity $P(\rho)=$ $\operatorname{Tr} \rho^{2}$, which satisfies $P(\rho) \leq 1$, with $P(\rho)=1$ iff $\rho$ is a pure state $\left(\rho^{2}=\rho\right)$. It is also directly related to the squared Hilbert-Schmidt distance to the maximally mixed state $I / d$ :

$$
\|\rho-I / d\|^{2}=\operatorname{Tr} \rho^{2}-1 / d=S_{2}(I / d)-S_{2}(\rho),
$$

where $\|O\|^{2}=\operatorname{Tr} O^{\dagger} O$ and $S_{2}(I / d)=1-1 / d$.

Similarly, the associated conditional entropy

$$
S_{2}\left(A \mid B_{\left\{\Pi_{j}\right\}}\right)=1-\sum_{j} p_{j} \operatorname{Tr} \rho_{A / \Pi_{j}}^{2},
$$

is trivially related with the average conditional purity $P\left(A \mid B_{\left\{\Pi_{j}\right\}}\right)=\sum_{j} p_{j} \operatorname{Tr} \rho_{A / \Pi_{j}}^{2}$, and determines the average squared distance to the maximally mixed state of $A$ :

$$
\sum_{j} p_{j}\left\|\rho_{A / \Pi_{j}}-I_{A} / d_{A}\right\|^{2}=S_{2}\left(I_{A} / d_{A}\right)-S_{2}\left(A \mid B_{\left\{\Pi_{j}\right\}}\right) .
$$

The ensuing $I_{2}(A \mid B)$ represents the average increase of the purity of $A$ due to the local measurement at $B$, and can be also interpreted as the average squared distance between the original and the post-measurement state of A:

$$
\begin{aligned}
I_{2}\left(A \mid B_{\left\{\Pi_{j}\right\}}\right) & =S_{2}(A)-S_{2}\left(A \mid B_{\left\{\Pi_{j}\right\}}\right) \\
& =\sum_{j} p_{j} \operatorname{Tr} \rho_{A / \Pi_{j}}^{2}-\operatorname{Tr} \rho_{A}^{2} \\
& =\sum_{j} p_{j}\left\|\rho_{A}-\rho_{A / \Pi_{j}}\right\|^{2},
\end{aligned}
$$

where we used Eq. (2). We may also define, through $I_{2}$ and $S_{2}$, the purity gain ratio

$$
R_{2}\left(A \mid B_{\left\{\Pi_{j}\right\}}\right)=1+\frac{I_{2}\left(A \mid B_{\left\{\Pi_{j}\right\}}\right)}{1-S_{2}(A)}=\frac{\sum_{j} p_{j} \operatorname{Tr} \rho_{A / \Pi_{j}}^{2}}{\operatorname{Tr} \rho_{A}^{2}},
$$

which satisfies $1 \leq R_{2}\left(A \mid B_{\left\{\Pi_{j}\right\}}\right) \leq d_{A}$. Such ratio remains unaltered if an ancilla $C$ at $A$ is added $\left(\rho_{A B} \rightarrow\right.$ $\left.\rho_{C} \otimes \rho_{A B}\right)$.

If $\rho$ is sufficiently close to the maximally mixed state $I / d$, Eq. (30) entails that all entropies $S_{f}(\rho)$ (with $\left.f^{\prime \prime}(p)<0 \forall p\right)$ become in this limit linear functions of $S_{2}(\rho)$ : A second order expansion of $S_{f}(\rho)$ around $\rho=I / d$ leads to

$$
\begin{aligned}
S_{f}(\rho)-S_{f}(I / d) & \approx \frac{1}{2} f^{\prime \prime}\left(\frac{1}{d}\right)\|\rho-I / d\|^{2} \\
& =\frac{1}{2}\left|f^{\prime \prime}\left(\frac{1}{d}\right)\right|\left[S_{2}(\rho)-S_{2}(I / d)\right] .
\end{aligned}
$$

Hence, in the vicinity of maximal mixedness, all entropies $S_{f}(\rho)$ (with $\left.f^{\prime \prime}(1 / d)<0\right)$, including of course the von
Neumann entropy $S(\rho)$, are determined by $S_{2}(\rho)$. In this limit $\rho_{A / \Pi_{j}}$ is also close to $I_{A} / d_{A} \forall \Pi_{j}$ and hence,

$$
\begin{aligned}
S_{f}\left(A \mid B_{\left\{\Pi_{j}\right\}}\right) \approx & S_{f}\left(I_{A} / d_{A}\right)+ \\
& \left.\frac{1}{2}\left|f^{\prime \prime}\left(\frac{1}{d_{A}}\right)\right|\left[S_{2}\left(A \mid B_{\left\{\Pi_{j}\right\}}\right)-S_{2}\left(I_{A} / d_{A}\right\rangle\right\}\right]
\end{aligned}
$$

indicating that all conditional entropies $S_{f}\left(A \mid B_{\left\{\Pi_{j}\right\}}\right)$ (with $\left.f^{\prime \prime}\left(1 / d_{A}\right)<0\right)$ also become functions of the $S_{2}$ conditional entropy. The measurement minimizing $S_{2}\left(A \mid B_{\left\{\Pi_{j}\right\}}\right)$ becomes then universal in this limit, i.e., it will also minimize all other $S_{f}\left(A \mid B_{\left\{\Pi_{j}\right\}}\right)$.

We note here that the geometric discord [8, 16] is defined as the minimum squared Hilbert-Schmidt distance from $\rho$ to a classically correlated state $\rho_{c}$ of the form (20), and is equivalent to the minimum increase of the $S_{2}$ entropy of the global state due to an unread projective measurement at $B$ 17]:

$D_{2}(A \mid B)=\operatorname{Min}_{\rho_{c}}\left\|\rho-\rho_{c}\right\|^{2}=\operatorname{Min}_{\left\{\Pi_{j}\right\}} S_{2}\left(\sum_{j} \Pi_{j} \rho \Pi_{j}\right)-S_{2}(\rho)$,

where again $\Pi_{j}=I_{A} \otimes \Pi_{j}^{B}$. In contrast with $S_{2}(A \mid B)$, the geometric discord looks for the closest average global post-measurement state $\sum_{j} \Pi_{j} \rho \Pi_{j}$. This will lead to significant differences in the minimizing measurement for certain states, as discussed in sec. 4 .

\section{B. Explicit expressions}

The obvious advantage of $S_{2}(\rho)$ over other entropies is that its evaluation does not require the knowledge of the eigenvalues of $\rho$. Convenient expressions in a system with Hilbert space dimension $d$ can be obtained just by considering a complete orthogonal set of hermitian operators $(I, \boldsymbol{\sigma})$, with $\boldsymbol{\sigma}=\left(\sigma_{1}, \ldots, \sigma_{d^{2}-1}\right)$ satisfying

$$
\operatorname{Tr} \sigma_{i}=0, \quad \operatorname{Tr} \sigma_{i} \sigma_{j}=d \delta_{i j}
$$

For a single qubit $\boldsymbol{\sigma}$ are the Pauli operators. A general state can then be written as

$$
\rho=(I+\boldsymbol{r} \cdot \boldsymbol{\sigma}) / d, \quad \boldsymbol{r}=\operatorname{Tr} \rho \boldsymbol{\sigma}=\langle\boldsymbol{\sigma}\rangle,
$$

and the quadratic entropy 29) becomes

$$
S_{2}(\rho)=1-\left(1+|\boldsymbol{r}|^{2}\right) / d .
$$

For a pure state $\rho^{2}=\rho,|\boldsymbol{r}|^{2}=d-1$ and $S_{2}(\rho)=0$.

In the case of a bipartite system $A+B$, we may rewrite Eq. (40) as

$$
\rho=\left[I+\boldsymbol{r}_{A} \cdot \boldsymbol{\sigma}_{A} \otimes I_{B}+I_{A} \otimes \boldsymbol{r}_{B} \cdot \boldsymbol{\sigma}_{B}+\boldsymbol{\sigma}_{A}^{t} J \otimes \boldsymbol{\sigma}_{B}\right] / d
$$

where $\boldsymbol{r}_{A}=\left\langle\boldsymbol{\sigma}_{A}\right\rangle, \boldsymbol{r}_{B}=\left\langle\boldsymbol{\sigma}_{B}\right\rangle$ and $J=\left\langle\boldsymbol{\sigma}_{A} \otimes \boldsymbol{\sigma}_{B}^{t}\right\rangle$ is a $\left(d_{A}^{2}-1\right) \times\left(d_{B}^{2}-1\right)$ matrix of elements $J_{i j}=\left\langle\sigma_{A i} \otimes \sigma_{B j}\right\rangle$. The reduced states are $\rho_{\alpha}=\left(I_{\alpha}+\boldsymbol{r}_{\alpha} \cdot \boldsymbol{\sigma}_{\alpha}\right) / d_{\alpha}, \alpha=A, B$.

A measurement $M_{B}$ based on the operators (11) can be characterized by the vectors

$$
\boldsymbol{k}_{j}=\operatorname{Tr}_{B}\left(\boldsymbol{\sigma}_{B}\left|j_{B}\right\rangle\left\langle j_{B}\right|\right),
$$


such that $\Pi_{j}^{B}=r_{j}\left(I_{B}+\boldsymbol{k}_{j} \cdot \boldsymbol{\sigma}_{B}\right) / d_{B}$. These vectors satisfy $\left|\boldsymbol{k}_{j}\right|^{2}=d_{B}-1$ and

$$
\sum_{j} r_{j} \boldsymbol{k}_{j}=\mathbf{0}
$$

since $\sum_{j} \Pi_{j}^{B}=I_{B}$. The probability of outcome $j$ and the ensuing state $\rho_{A / \Pi_{j}}$ are then

$p_{j}=\frac{r_{j}}{d_{B}}\left(1+\boldsymbol{r}_{B} \cdot \boldsymbol{k}_{j}\right), \quad \rho_{A / \Pi_{j}}=\frac{1}{d_{A}}\left[I_{A}+\frac{\left(\boldsymbol{r}_{A}+J \boldsymbol{k}_{j}\right) \cdot \boldsymbol{\sigma}_{A}}{1+\boldsymbol{r}_{B} \cdot \boldsymbol{k}_{j}}\right]$,

which involve just the components of $\boldsymbol{r}_{B}$ and $J$ along $\boldsymbol{k}_{j}$. Eqs. (41)-45) lead then to

$$
\begin{aligned}
S_{2}\left(A \mid B_{\left\{\Pi_{j}\right\}}\right) & =1-\frac{1}{d_{A}}\left[1+\sum_{j} p_{j} \frac{\left|\boldsymbol{r}_{A}+J \boldsymbol{k}_{j}\right|^{2}}{\left(1+\boldsymbol{r}_{B} \cdot \boldsymbol{k}_{j}\right)^{2}}\right] \\
& =S_{2}(A)-\frac{1}{d} \sum_{j} r_{j} \frac{\boldsymbol{k}_{j}^{t} C^{t} C \boldsymbol{k}_{j}}{1+\boldsymbol{r}_{B} \cdot \boldsymbol{k}_{j}},
\end{aligned}
$$

where $S_{2}(A)=S_{2}\left(\rho_{A}\right)=1-\left(1+\left|\boldsymbol{r}_{A}\right|^{2}\right) / d_{A}$ and $\boldsymbol{k}_{j}^{t} C^{t} C \boldsymbol{k}_{j}=\left|C \boldsymbol{k}_{j}\right|^{2}$, with

$$
C=J-\boldsymbol{r}_{A} \boldsymbol{r}_{B}^{t}=\left\langle\boldsymbol{\sigma}_{A} \otimes \boldsymbol{\sigma}_{B}^{t}\right\rangle-\left\langle\boldsymbol{\sigma}_{A}\right\rangle\left\langle\boldsymbol{\sigma}_{B}^{t}\right\rangle,
$$

the correlation matrix, of elements $C_{i j}=\left\langle\sigma_{A i} \otimes \sigma_{B j}\right\rangle-$ $\left\langle\sigma_{A i}\right\rangle\left\langle\sigma_{B j}\right\rangle\left(C=0\right.$ iff $\left.\rho=\rho_{A} \otimes \rho_{B}\right)$. The second term in (46) is just the quadratic information gain (i.e., purity increase) (33):

$$
I_{2}\left(A \mid B_{\left\{\Pi_{j}\right\}}\right)=\frac{1}{d} \sum_{j} r_{j} \frac{\boldsymbol{k}_{j}^{t} C^{t} C \boldsymbol{k}_{j}}{1+\boldsymbol{r}_{B} \cdot \boldsymbol{k}_{j}} .
$$

It is then determined by $\boldsymbol{r}_{B}$ and the $\left(d_{B}^{2}-1\right) \times\left(d_{B}^{2}-1\right)$ positive semidefinite matrix $C^{t} C$. We finally note that we may also express Eqs. (42) and (45) in terms of the correlation matrix $C$ (rather than $J$ ) as

$$
\begin{aligned}
\rho & =\rho_{A} \otimes \rho_{B}+\boldsymbol{\sigma}_{A}^{t} C \otimes \boldsymbol{\sigma}_{B} / d, \\
\rho_{A / \Pi_{j}} & =\rho_{A}+\frac{\boldsymbol{\sigma}_{A}^{t} C \boldsymbol{k}_{j}}{d_{A}\left(1+\boldsymbol{r}_{B} \cdot \boldsymbol{k}_{j}\right)},
\end{aligned}
$$

with $\left\|\rho-\rho_{A} \otimes \rho_{B}\right\|^{2}=\operatorname{Tr}\left[C^{t} C\right] / d=\|C\|^{2} / d$.

\section{The qudit-qubit case}

We now show that when $B$ is a single qubit, an analytic expression for the minimum $S_{2}$ conditional entropy (i.e., for the maximum conditional purity of $A$ ) amongst projective local measurements on $B$ can be obtained for any dimension $d_{A}$ of $A\left(\mathbb{C}^{d_{A}} \otimes \mathbb{C}^{2}\right.$ system $)$ and any initial state $\rho$. Here we can take $\boldsymbol{\sigma}_{B}$ as the Pauli operators, and $\boldsymbol{k}_{j}$ become unit vectors. For a projective spin measurement along direction $\boldsymbol{k}(|\boldsymbol{k}|=1)$, we have $j=1,2$, with $r_{j}=1, \boldsymbol{k}_{1}=-\boldsymbol{k}_{2}=\boldsymbol{k}$, and Eq. (48) becomes

$$
I_{2}\left(A \mid B_{\boldsymbol{k}}\right)=\frac{1}{d_{A}} \frac{\boldsymbol{k}^{t} C^{t} C \boldsymbol{k}}{1-\left(\boldsymbol{r}_{B} \cdot \boldsymbol{k}\right)^{2}}=\frac{1}{d_{A}} \frac{\boldsymbol{k}^{t} C^{t} C \boldsymbol{k}}{\boldsymbol{k}^{t} N_{B} \boldsymbol{k}},
$$

where $N_{B}$ is the $3 \times 3$ positive semidefinite matrix

$$
N_{B}=I_{3}-\boldsymbol{r}_{B} \boldsymbol{r}_{B}^{t} .
$$

The last expression in (50) is a ratio of quadratic forms and is then independent of the length of $\boldsymbol{k}$. Its maximum can therefore be obtained diagonalizing the $3 \times 3$ matrix $C^{t} C$ with the metric $N_{B}$ : Setting $\boldsymbol{k}=N_{B}^{-1 / 2} \tilde{\boldsymbol{k}}$, with $\tilde{\boldsymbol{k}}^{t} \tilde{\boldsymbol{k}}=1$, we have

$$
\frac{\boldsymbol{k}^{t} C^{t} C \boldsymbol{k}}{\boldsymbol{k}^{t} N_{B} \boldsymbol{k}}=\tilde{\boldsymbol{k}}^{t} \tilde{C}^{t} \tilde{C} \tilde{\boldsymbol{k}} \leq \lambda_{\max }
$$

where $\tilde{C}=C N_{B}^{-1 / 2}$ and $\lambda_{\max }$ is the maximum eigenvalue of $\tilde{C}^{t} \tilde{C}$, the maximum reached when $\tilde{\boldsymbol{k}}$ is the associated normalized eigenvector. The eigenvalue equation $\tilde{C}^{t} \tilde{C} \tilde{\boldsymbol{k}}=\lambda \tilde{\boldsymbol{k}}$ is just the eigenvalue equation for $C^{t} C$ with metric $N_{B}$,

$$
C^{t} C \boldsymbol{k}=\lambda N_{B} \boldsymbol{k},
$$

so that $\lambda_{\max }$ is the largest root of the equation

$$
\operatorname{Det}\left[C^{t} C-\lambda N_{B}\right]=0,
$$

with $\boldsymbol{k}$ the associated eigenvector. In other words, $\sqrt{\lambda_{\max }}$ is the maximum singular value of the matrix $C$ with metric $N_{B}$. The ensuing minimum conditional entropy and maximum information gain (uncertainty reduction) for projective measurements are then

$$
\begin{aligned}
& S_{2}(A \mid B)=\operatorname{Min}_{\boldsymbol{k}} S_{2}\left(A \mid B_{\boldsymbol{k}}\right)=S_{2}(A)-\lambda_{\max } / d_{A}, \\
& I_{2}(A \mid B)=\operatorname{Max}_{\boldsymbol{k}} I_{2}\left(A \mid B_{\boldsymbol{k}}\right)=\lambda_{\max } / d_{A} .
\end{aligned}
$$

If $\boldsymbol{r}_{B}=\mathbf{0}, N_{B}=I_{3}$ and $\lambda_{\max }$ is just the maximum eigenvalue of $C^{t} C$. On the other hand, if $\left|\boldsymbol{r}_{B}\right|=1, \rho$ is a product state and $\boldsymbol{k}^{t} C^{t} C \boldsymbol{k}=0$ vanishes $\forall \boldsymbol{k}$.

For instance, the classically correlated state (20) corresponds, choosing the $z$ axis in $B$ such that $\tilde{\Pi}_{ \pm \boldsymbol{k}}^{B}=$ $\frac{1}{2}\left(I_{B} \pm \sigma_{z}\right)$, to $\left(\boldsymbol{r}_{B}\right)_{\nu}=\delta_{\nu z} r_{B}, J_{\mu \nu}=\delta_{\nu z} J_{\mu z}$, implying $\left(C^{t} C\right)_{\nu \nu^{\prime}}=\delta_{\nu \nu^{\prime}} \delta_{\nu z}\left|\boldsymbol{J}-r_{B} \boldsymbol{r}_{A}\right|^{2}$, with $\boldsymbol{J}$ the vector of components $J_{\mu z}$. Hence,

$$
\frac{\boldsymbol{k}^{t} C^{t} C \boldsymbol{k}}{\boldsymbol{k}^{t} N_{B} \boldsymbol{k}} \leq \lambda_{\max }=\frac{\left|\boldsymbol{J}-r_{B} \boldsymbol{r}_{A}\right|^{2}}{1-r_{B}^{2}},
$$

being verified that the maximum is reached for $\boldsymbol{k}$ along $z$, i.e., for a spin measurement along $\boldsymbol{r}_{B}$ (basis of eigenstates of $\left.\rho_{B}\right)$. For a general state however, the minimizing direction may differ from $\boldsymbol{r}_{B}$ and follow the main correlation in $C^{t} C$.

If $A$ is also a qubit $\left(d_{A}=2\right)$, it is convenient to use $S_{2}(\rho)=2\left(1-\operatorname{Tr} \rho^{2}\right)$ in previous equations, i.e. $\frac{1}{d_{A}} \rightarrow 1$ in Eqs. (50)-(56), such that $S_{2}\left(\rho_{A}\right)=1$ if $\rho_{A}$ is maximally mixed. Such rescaled entropy is still a lower bound to the $a=2$ von Neumann entropy $S(\rho)=-\operatorname{Tr} \rho \log _{2} \rho$ (see A). In such a case, if $\rho$ is of rank 2, it can be purified by adding a third qubit $C$, being then verified that $S_{2}(A \mid B)$ coincides with the squared concurrence 47] between $A$ 
and $C$, since such quantity reduces for pure two-qubit states to the present rescaled $S_{2}$ entropy of any of the subsystems, and coincides with its convex roof extension $E_{2}(A, C)$ for mixed two qubit states 41 .

We remark finally that for a qudit-qubit state, the (minimum) geometric discord (38) is determined by the largest eigenvalue of a different $3 \times 3$ matrix [8, 16]:

$$
D_{2}(A \mid B)=\frac{1}{d}\left(\left|\boldsymbol{r}_{B}\right|^{2}+\|J\|^{2}-\tilde{\lambda}_{\max }\right),
$$

where $\tilde{\lambda}_{\max }$ is the largest eigenvalue of $M_{2}=\boldsymbol{r}_{B} \boldsymbol{r}_{B}^{t}+$ $J^{t} J$. This matrix depends then on $J$ rather than the correlation $C$, coinciding with $C^{t} C$ just when $r_{B}=0$.

\section{APPLICATION}

\section{A. $\mathrm{X}$ states}

Let us now consider a two-qubit system. Through its singular value decomposition, the now $3 \times 3$ matrix $J$ can be always brought to the diagonal form $J_{\mu \nu}=\delta_{\mu \nu} J_{\mu}$ by appropriately choosing the local $x, y, z$ axes. If $\boldsymbol{r}_{A}$ and $\boldsymbol{r}_{B}$ are directed along the same principal axes of $J$, which we shall denote as $z$, we obtain an $X$ state [30],

$$
\begin{aligned}
\rho & =\frac{1}{4}\left(I+r_{A} \sigma_{z} \otimes I_{2}+r_{B} I_{2} \otimes \sigma_{z}+\sum_{\mu=x, y, z} J_{\mu} \sigma_{\mu} \otimes \sigma_{\mu}\right) \\
& =\left(\begin{array}{cccc}
p_{+} & 0 & 0 & \alpha_{-} \\
0 & q_{+} & \alpha_{+} & 0 \\
0 & \alpha_{+} & q_{-} & 0 \\
\alpha_{-} & 0 & 0 & p_{-}
\end{array}\right), \quad \begin{array}{c}
p_{ \pm}=\frac{1 \pm\left(r_{A}+r_{B}\right)+J_{z}}{4}=\frac{1 \pm\left(r_{A}-r_{B}\right)-J_{z}}{J_{x} J_{y}} \\
\alpha_{ \pm}=\frac{J_{x}}{4}
\end{array}
\end{aligned}
$$

where Eq. (60) is its standard basis representation. This state commutes with the $z$ parity $P_{z}=\sigma_{z} \otimes \sigma_{z}$. Accordingly, reduced states of arbitrary spin pairs in the thermal state or in any non-degenerate eigenstate of any spin $1 / 2$ array with $X Y$ or $X Y Z$ Heisenberg couplings of arbitrary range in a field along $z$, are of the present form [31], as the corresponding Hamiltonian (see Eq. (711) commutes with the total $z$ parity.

The ensuing matrices $C$ and $N_{B}$ are simultaneously diagonal,

$$
C=\left(\begin{array}{ccc}
J_{x} & 0 & 0 \\
0 & J_{y} & 0 \\
0 & 0 & J_{z}-r_{A} r_{B}
\end{array}\right), N_{B}=\left(\begin{array}{ccc}
1 & 0 & 0 \\
0 & 1 & 0 \\
0 & 0 & r_{B}^{2}
\end{array}\right)
$$

Hence, the minimum conditional entropy $S_{2}(A \mid B)$ among projective measurements will be obtained for a measurement along one of the principal axes $x, y, z$. We then obtain

$$
\begin{aligned}
S_{2}(A \mid B) & =1-\left|\boldsymbol{r}_{A}\right|^{2}-I_{2}(A \mid B), \\
I_{2}(A \mid B) & =\operatorname{Max}_{\boldsymbol{k}} \frac{\boldsymbol{k}^{t} C^{t} C \boldsymbol{k}}{\boldsymbol{k}^{t} N_{B} \boldsymbol{k}} \\
& =\operatorname{Max}\left[J_{x}^{2}, J_{y}^{2}, \frac{\left(J_{z}-r_{A} r_{B}\right)^{2}}{1-r_{B}^{2}}\right],
\end{aligned}
$$

for $S_{2}(\rho)=2\left(1-\operatorname{Tr} \rho^{2}\right)$, implying a $z \rightarrow x$ or $z \rightarrow y$ transition in the direction of the minimizing measurement as $J_{x}^{2}$ or $J_{y}^{2}$ increase across $\lambda_{z}=\left(J_{z}-r_{A} r_{B}\right)^{2} /\left(1-r_{B}^{2}\right)$.

Such direction is then determined essentially by the main correlation in $C^{t} C$. This provides a conceptual basis for the results of [33] related with the minimizing measurement of the quantum discord for $X$ states, which also follow the main correlation. This direction can then differ significantly from that minimizing the geometric discord (38) - (58). For the state (59) we obtain [16, 17] (Eq. (58) )

$$
D_{2}(A \mid B)=\frac{1}{2}\left\{r_{B}^{2}+\|J\|^{2}-\operatorname{Max}\left[J_{x}^{2}, J_{y}^{2}, J_{z}^{2}+r_{B}^{2}\right]\right\}
$$

entailing a $z \rightarrow x$ or $z \rightarrow y$ transition only as $J_{x}^{2}$ or $J_{y}^{2}$ increase across $J_{z}^{2}+r_{B}^{2}$. Coincidence between both minimizing measurements can then be ensured just for $r_{B}=0$, i.e., $\rho_{B}$ maximally mixed, where the minimizing $\boldsymbol{k}$ is along the axis with the largest $\left|J_{\mu}\right|$ for both $S_{2}(A \mid B)$ and $D_{2}(A \mid B)$.

For a general entropy $S_{f}$, the conditional entropy is (Eq. 45),

$S_{f}\left(A \mid B_{\boldsymbol{k}}\right)=\sum_{\mu, \nu= \pm 1} \frac{1+\nu \boldsymbol{r}_{B} \cdot \boldsymbol{k}}{2} f\left[\frac{1}{2}\left(1+\mu\left|\boldsymbol{r}_{A}+\frac{\nu C \boldsymbol{k}}{1+\nu \boldsymbol{r}_{B} \cdot \boldsymbol{k}}\right|\right)\right]$.

It is verified that for an $X$ state, measurements along any of the principal axes of $J$ (i.e., $x, y, z$ ) are always stationary $\left(\delta S_{f}\left(A \mid B_{\boldsymbol{k}}\right)=0\right.$ up to first order in $\left.\delta \boldsymbol{k}\right)$, i.e., candidates for minimizing (64), although other directions cannot be discarded (typically in the transitional region between the $z$ and $x$ or $y$ regimes). On the other hand, for two qubit states with maximally mixed marginals, which can be written as $X$ states with $r_{A}=r_{B}=0$, it is seen from (62) and 64 that the minimizing measurement is along the axis with the largest $\left|J_{\mu}\right|$, i.e., $\boldsymbol{k}$ along the largest eigenvalue of $J^{t} J=C^{t} C$, for any entropy $S_{f}$ (universal minimum).

We finally mention that the geometric discord $D_{2}(A \mid B)$ was shown in 48 to be an upper bound to the square of the negativity $\mathcal{N}(\rho)$, a computable entanglement monotone 49, given for two qubits by $\mathcal{N}(\rho)=\operatorname{Tr}\left|\rho^{T_{B}}\right|-1$, with $\rho^{t_{B}}$ the partial transpose, both coinciding for $\rho$ pure. For $X$ states we obtain here a similar relation between $I_{2}(A \mid B)$ and the squared concurrence $\mathcal{C}^{2}(\rho)$, with both also coinciding when $\rho$ is pure: For the state (60), the concurrence [47 is $\mathcal{C}(\rho)=2 \operatorname{Max}\left[\left|\alpha_{+}\right|-\sqrt{p_{+} p_{-}},\left|\alpha_{-}\right|-\sqrt{q_{+} q_{-}}, 0\right]$, imply$\operatorname{ing} \mathcal{C}(\rho) \leq 2 \operatorname{Max}\left[\left|\alpha_{+}\right|,\left|\alpha_{-}\right|\right]$and hence, since $\left|\alpha_{ \pm}\right| \leq$ $\operatorname{Max}\left[\left|J_{x}\right|,\left|J_{y}\right|\right] / 2$,

$$
\mathcal{C}^{2}(\rho) \leq \operatorname{Max}\left[J_{x}^{2}, J_{y}^{2}\right] \leq I_{2}(A \mid B) .
$$

\section{B. Mixture of a pure state with the maximally mixed state}

As a specific example of (59), we consider the mixture (25) in the two qubit case. By suitable choosing the local 
axes, we may always write it as

$\rho=w|\Psi\rangle\left\langle\Psi\left|+(1-w) I_{4} / 4, \quad\right| \Psi\right\rangle=\sqrt{q}|00\rangle+\sqrt{1-q}|11\rangle$,

which corresponds to an $X$ state with

$r_{A}=r_{B}=w(2 q-1), J_{x}=-J_{y}=2 w \sqrt{q(1-q)}, J_{z}=w$.

It is then verified that $\frac{\left(J_{z}-r_{A} r_{B}\right)^{2}}{1-r_{B}^{2}}-J_{x}^{2}=$ $\frac{w^{2}(1-w)^{2}(1-2 q)^{2}}{1-w^{2}(1-2 q)^{2}} \geq 0$, implying that $S_{2}\left(A \mid B_{\boldsymbol{k}}\right)$ is minimized by a measurement along $z$ (basis of eigenstates of $\rho_{B}$ ), in agreement with the universal minimum for this state. It is also seen that for $w=1$ ( $\rho$ pure), $w=0$ ( $\rho$ maximally mixed) or $q=1 / 2(|\Psi\rangle$ maximally entangled) the previous difference vanishes, indicating that all directions $\boldsymbol{k}$ lead to the same result, in agreement with previous considerations. In any case we obtain, for $S_{2}(\rho)=2\left(1-\operatorname{Tr} \rho^{2}\right)$,

$$
\begin{aligned}
S_{2}(A \mid B) & =\frac{(1-w)\left(1+w-2 w^{2}(1-2 q)^{2}\right)}{1-w^{2}(1-2 q)^{2}}, \\
I_{2}(A \mid B) & =\frac{w^{2}\left(1-w(1-2 q)^{2}\right)^{2}}{1-w^{2}(1-2 q)^{2}},
\end{aligned}
$$

with $S_{2}(A)=1-w^{2}(1-2 q)^{2}$. It is verified that Eq. [67] is a strictly concave decreasing function of $w$ at fixed $q \in[0,1]$, and a strictly concave function of $q$ if $w \in(0,1)$, reaching its maximum at $q=1 / 2$ (Bell state). Notice that $(1-2 q)^{2}=1-\mathcal{C}^{2}(|\Psi\rangle)$, with $\mathcal{C}(|\Psi\rangle)=2 \sqrt{q(1-q)}$ the concurrence [47 of $|\Psi\rangle$, so that Eq. [67] is, for $w \in(0,1)$, an increasing function of $\mathcal{C}(|\Psi\rangle)$, i.e. of entanglement, as previously ascertained. The bound (65) is also verified $(\mathcal{C}(\rho)=\operatorname{Max}[w \mathcal{C}(|\Psi\rangle)-(1-w) / 2,0])$.

Eq. (68) is also a strictly concave function of $q$ if $w \in(0,1]$, maximum at $q=1 / 2$, i.e., an increasing function of the concurrence $\mathcal{C}(|\Psi\rangle)$. In contrast, Eq. 68 is not necessarily an increasing function of $w$. Its behavior with $w$ can be non-monotonous if $|\Psi\rangle$ is separable or almost separable ( $q$ small or close to 1$)$, as shown in Fig. 1, where results for the von Neumann based $\left(S(\rho)=-\operatorname{Tr} \rho \log _{2} \rho\right)$ conditional entropy and information gain are also depicted. Such behavior is universal, i.e., present for any $S_{f}$ : When $|\Psi\rangle$ is separable, noise induces a non-zero value of $I_{f}(A \mid B)$, since $\rho$ ceases to be a product state for $w \in(0,1)$. As seen in Fig. 1 the qualitative behavior of the minimum linear and von Neumann conditional entropies is entirely similar, and the same holds for the ensuing maximum $I_{f}(A \mid B)$. Nonetheless, while $S_{2}(A \mid B) \leq S(A \mid B)$, there is in general no fixed order relation between $I_{2}(A \mid B)$ and $I(A \mid B)$.

\section{Mixture of aligned states}

We now consider the two-qubit mixed state

$$
\rho=\frac{1}{2}(|\theta \theta\rangle\langle\theta \theta|+|-\theta \theta\rangle\langle-\theta-\theta|),
$$
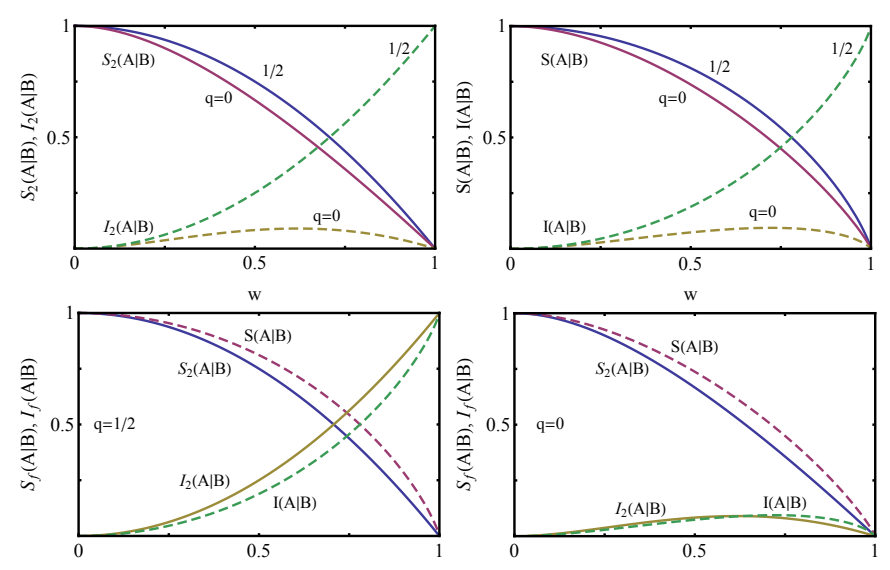

FIG. 1. (Color online) Top: Results for the quadratic (left) and von Neumann (right) minimum conditional entropy $S_{f}(A \mid B)$ (solid lines) and maximum information gain (or uncertainty reduction) $I_{f}(A \mid B)$ (dashed lines), after a measurement at $B$ in the mixture (66) for the maximally entangled $(q=1 / 2)$ and separable $(q=0)$ cases. All $S_{f}(A \mid B)$ are concave decreasing functions of $w$, vanishing at the pure limit $w=1$. Bottom: Comparison between quadratic (solid lines) and von Neumann (dashed lines) results for $q=1 / 2$ (left) and $q=0$ (right). It is verified that $S_{2}(A \mid B) \leq S(A \mid B) \forall w, q$.

where $|\theta\rangle=\exp \left[-i \theta \sigma_{y} / 2\right]|0\rangle=\cos \frac{\theta}{2}|0\rangle+\sin \frac{\theta}{2}|1\rangle$ is the state with the spin forming an angle $\theta$ with the $z$ axis. This separable state represents, roughly, the reduced state of a spin $1 / 2$ pair in the exact definite parity ground state of a ferromagnetic $X Y$ chain for fields $|B|<B_{c}$ if $\cos \theta=B / B_{c}$ 31]. Moreover, for not too small chains it is the exact state of the pair in the immediate vicinity of the factorizing field [31, 50, 51]. Eq. (69) is an $X$ state with

$$
r_{A}=r_{B}=\cos \theta, \quad J_{z}=\cos ^{2} \theta, \quad J_{x}=\sin ^{2} \theta, \quad J_{y}=0 .
$$

Hence, there is no correlation along $z\left(J_{z}=r_{A} r_{B}\right.$, implying $C_{z}=0$ ) but there is a finite correlation along $x$ $\left(C_{x}=J_{x}^{2}\right)$. We then obtain the remarkable result that $S_{2}\left(A \mid B_{\boldsymbol{k}}\right)$ is minimized for $\boldsymbol{k}$ along $x \forall \theta \in(0, \pi / 2]$, leading to

$$
\begin{aligned}
& S_{2}(A \mid B)=1-\cos ^{2} \theta-\sin ^{4} \theta=\frac{1}{4} \sin ^{2} 2 \theta, \\
& I_{2}(A \mid B)=\sin ^{4} \theta .
\end{aligned}
$$

The minimum $S_{2}$ conditional entropy is then symmetric around $\theta=\pi / 4$, vanishing for $\theta=0$ (product state) and $\pi / 2$ (classically correlated state of the form (20) with $\rho_{A / \boldsymbol{k}}$ pure), whereas the maximum $I_{2}(A \mid B)$ increases with $\theta$ (Fig. 2), reaching its absolute maximum at $\theta=\pi / 2$. Hence, spin measurements along $z$ are not minimum for any $\theta>0$ (although the difference with (70) is $O\left(\theta^{4}\right)$ for $\left.\theta \rightarrow 0\right)$.

In the von Neumann case, the behavior of $S(A \mid B)$ and $I(A \mid B)$ is again completely similar to that of $S_{2}(A \mid B)$ and $I_{2}(A \mid B)$, as seen in Fig. 2] Moreover, the minimizing measurement is also for $\boldsymbol{k}$ along $x \forall \theta \in(0, \pi / 2]$ [17, 31, 

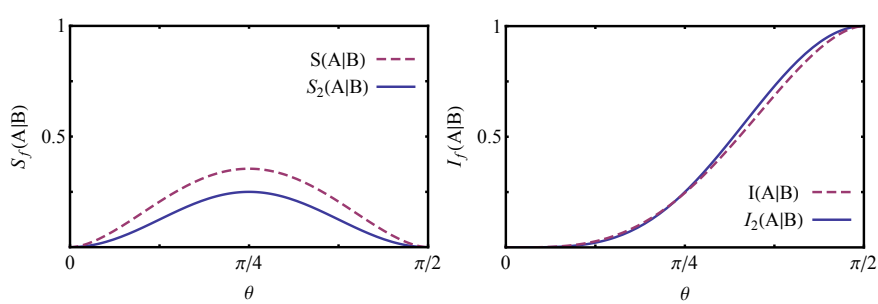

FIG. 2. (Color online) Results for the quadratic (solid lines) and von Neumann (dashed lines) minimum conditional entropy (left) and maximum information gain or uncertainty reduction (right) in the mixture of aligned states (69). Both entropies are minimized by a spin measurement along $x \forall$ $\theta \in(0, \pi / 2]$.

i.e., the same as that of the $S_{2}$ entropy $\forall \theta$. The $S_{2}$ results allow then to easily understand the minimizing measurement of the quantum discord for this state 31. In contrast, the geometric discord is minimized for $\boldsymbol{k}$ along $x$ only if $\theta>\theta_{c}$, with $\cos ^{2} \theta_{c}=\frac{1}{3}$, preferring $\boldsymbol{k}$ along $z$ if $\theta<\theta_{c}$ [17].

\section{Spin $1 / 2$ pairs in $X Y$ chains at strong transverse fields}

Let us finally consider a spin $1 / 2$ array with $X Y$ couplings in a strong transverse field, described by a Hamiltonian

$$
H=-B \sum_{i} \sigma_{i z}-\sum_{i<j}\left(J_{i j}^{x} \sigma_{i x} \sigma_{j x}+J_{i j}^{y} \sigma_{i y} \sigma_{j y}\right) .
$$

For sufficiently strong fields $B \gg\left|J_{i j}^{\mu}\right| \forall \mu, i, j$, the system is weakly coupled and the ground state is of the form

$$
|\Psi\rangle \approx|0\rangle+\sum_{i<j} \alpha_{i j}|i j\rangle
$$

at lowest non trivial order, where $|0\rangle=|0 \ldots 0\rangle$ denotes the state with all spins aligned along the field $(+z),|i j\rangle=$ $\sigma_{i-} \sigma_{j-}|0\rangle$ and $\alpha_{i j} \approx\left(J_{i j}^{x}-J_{i j}^{y}\right) /(2 B)$. The reduced state of a pair $i, j$ is therefore an $X$ state with, at lowest nonzero order (we set $\alpha_{j i}=\alpha_{i j}$ ),

$$
\begin{aligned}
& \alpha_{-}=\alpha_{i j}, \quad p_{-}=\left|\alpha_{i j}\right|^{2}, \\
& \alpha_{+}=\sum_{k \neq i, j} \alpha_{i k} \bar{\alpha}_{k j}, \quad q_{ \pm}=\sum_{k \neq i, j}\left|\alpha_{j} k\right|^{2} .
\end{aligned}
$$

By suitably choosing the local states at sites $i, j$ we may set $\alpha_{ \pm}$real and positive. Hence, up to $O\left(|\alpha|^{2}\right)$ we obtain $r_{A, B}=1-2\left(\left|\alpha_{i j}\right|^{2}+q_{\mp}\right)$ (along $z$ ) and

$$
J_{x}=2\left(\sum_{k \neq i, j} \alpha_{i k} \bar{\alpha}_{k j} \pm \alpha_{i j}\right), \quad J_{z}-r_{A} r_{B} \approx 4\left|\alpha_{i j}\right|^{2} .
$$

Hence, for $\alpha_{i j} \neq 0$ (interacting pair), $C_{x x}$ is $O\left(\alpha_{i j}\right)$ whereas $C_{z z}$ is $O\left(\alpha_{i j}^{2}\right)$, entailing at lowest order a minimizing measurement along $x$ instead of $z$, as the correlation along $z$ is of higher order. The same behavior occurs with the minimizing measurement of the von Neumann conditional entropy and hence the quantum discord in this regime ( $\boldsymbol{k}$ along $x$ at strong fields 31,52 ). In contrast, that minimizing the geometric discord or the information deficit 9, 17] follows the main component of the state, and is therefore along the field direction $z$ for strong fields 52].

\section{CONCLUSIONS}

We have analyzed the main features of the conditional entropy associated to general concave entropic forms in bipartite quantum systems, determined by a measurement in one of the constituents. Its minimum among all local measurements determines the maximum average uncertainty reduction (generalized information gain) about $A$ that can be achieved by a measurement on $B$, and has the direct meaning of representing the associated entanglement of formation between $A$ and a purifying third system $C$. For some important classes of states as those of sections 2.3 and 2.4, the minimizing measurement is the same for all $S_{f}$ and can be analytically and identified, allowing a direct general evaluation of $E_{f}(A, C)$. This universality indicates that for such states there is clearly an unambiguous optimum local measurement leading to the lowest conditional mixedness at the unmeasured part, irrespective of the measure used for quantifying such mixedness.

For the general case, a main practical result of our manuscript is the analytic determination of this minimum for the linear entropy $S_{2}$ in a general qudit+qubit state with projective measurements. It can be expressed in terms of the largest eigenvalue of a simple $3 \times 3$ matrix, which represents the largest singular value of the correlation matrix $C$ with a metric $N_{B}$ determined by the measured part. This enables to easily identify the minimizing measurement, determined by the associated eigenvector, and understand its behavior. Conditional $S_{2}$ results have also a direct interpretation in terms of purity and average distances, and possess the importance of determining the universal behavior of all conditional entropies and the ensuing minimizing measurement in the vicinity of maximum mixedness.

In the specific examples considered, the minimizing measurements of the $S_{2}$ and von Neumann conditional entropies (and hence the quantum discord) were in fact coincident. The present results explain then the quite distinct response of this minimizing measurement to the onset of correlations (it follows the main correlation even if arbitrarily weak), in comparison with those minimizing the geometric discord or the one way information deficit, which follow instead the main component of the state 52]. Hence, the present formalism not only allows to identify universal features and optimize postmeasurement purities, but can also help to evaluate or estimate the quantum discord in more complex situations, as the minimizing measurements for the linear and 
von Neumann conditional entropies become coincident in some states and regimes, and can be expected to be close in typical situations.

The authors acknowledge support of CIC (RR) and CONICET (NG) of Argentina.

\section{Appendix A: Trace form generalized entropies}

Given a quantum state $\rho$ with spectral decomposition $\rho=\sum_{j} p_{j}|j\rangle\langle j|, j=1, \ldots, d\left(p_{j} \geq 0, \sum_{j} p_{j}=1\right)$, the "entropic" forms (see for instance 12, 35])

$$
S_{f}(\rho)=\operatorname{Tr} f(\rho)=\sum_{j} f\left(p_{j}\right),
$$

comply, for any strictly concave real function $f:[0,1] \rightarrow$ $\Re$ satisfying $f(0)=f(1)=0$, with all conventional entropy properties except additivity: i) $S_{f}(\rho) \geq 0$, with $S_{f}(\rho)=0$ iff $\rho$ is pure $\left(\rho^{2}=\rho\right)$, ii) $S_{f}(\rho)$ is maximum at the maximally mixed state $I_{d} / d$, with $S_{f}\left(I_{d} / d\right)=$ $d f(1 / d)$ an increasing function of $d$, iii) $S_{f}\left(U \rho U^{\dagger}\right)=$ $S_{f}(\rho) \forall$ unitary $U$ and iv) $S_{f}(\rho)$ is concave (Eq. 5) (if $\rho=\sum_{\alpha} q_{\alpha} \rho_{\alpha}, f\left(p_{j}\right)=f\left(\sum_{\alpha, j^{\prime}} q_{\alpha}\left|\left\langle j \mid j_{\alpha}^{\prime}\right\rangle\right|^{2} p_{j^{\prime}}^{\alpha}\right) \geq$ $\sum_{j,{ }^{\prime} \alpha} q_{\alpha}\left|\left\langle j \mid j_{\alpha}^{\prime}\right\rangle\right|^{2} f\left(p_{j^{\prime}}^{\alpha}\right)$, which leads to (5) after summing over $j$ ).

Concavity implies ii) and, moreover, the majorization 12, 53. property [17, 54.

$$
\rho \prec \rho^{\prime} \Rightarrow S_{f}(\rho) \geq S_{f}\left(\rho^{\prime}\right),
$$

where $\rho \prec \rho^{\prime}$ ( $\rho$ more mixed than $\rho^{\prime}$ ) means $\sum_{j=1}^{i} p_{j} \leq$ $\sum_{j=1}^{i} p_{j}^{\prime}$ for $i=1, \ldots, d-1$, with $p_{j}, p_{j}^{\prime}$ denoting here the eigenvalues of $\rho$ and $\rho^{\prime}$ sorted in decreasing order (and completed with 0's if dimensions differ). Eq. A22 provides the conceptual basis for considering any such $S_{f}$ a generalized uncertainty measure or entropic form. Furthermore, while the converse of A2 does not necessarily hold if valid for some particular $S_{f}$ (majorization is stronger than a single entropic inequality), it does hold if valid $\forall S_{f}$ of the form (A1): $S_{f}(\rho) \geq S_{f}\left(\rho^{\prime}\right) \forall S_{f} \Rightarrow$ $\rho \prec \rho^{\prime}$ 54].

The Tsallis entropy [4] $S_{q}(\rho)=\left(1-\operatorname{Tr} \rho^{q}\right) /(q-1)$, $q>0$, corresponds to $f(\rho)=\left(\rho-\rho^{q}\right) /(q-1)$ in A1. It reduces to the quadratic entropy (29) for $q=2$ and to the von Neumann entropy (with $a=e$ ) for $q \rightarrow 1$. We may also set $S_{q}(\rho)=\left(1-\operatorname{Tr} \rho^{q}\right) /\left(1-2^{1-q}\right)$, such that $S_{q}(\rho)=1$ for a maximally mixed single qubit state $\rho=I_{2} / 2$, in which case $S_{2}(\rho)=2\left(1-\operatorname{Tr} \rho^{2}\right)$ and $S_{q}(\rho) \rightarrow$ $-\operatorname{Tr} \rho \log _{2} \rho$ for $q \rightarrow 1$. For this scaling it is still verified that $S_{2}(\rho) \leq S(\rho)$ for any single qubit state, coinciding just for $\rho$ pure or maximally mixed (for any single qubit state, $S_{q}(\rho) \leq S(\rho)$ for $1<q<q_{1} \approx 4.718$ with this scaling).

For two classical random variables $A, B$ described by a joint probability distribution $p_{i j}=p(A=i, B=j)$, we may define a generalized conditional entropy $S_{f}(A \mid B)$ as

$$
S_{f}(A \mid B)=\sum_{j} p_{j} S_{f}(A \mid B=j)=\sum_{i, j} p_{j} f\left(p_{i j} / p_{j}\right),
$$

where $p_{j}=p(B=j)=\sum_{i} p_{i j}$. This quantity measures the average uncertainty about $A$ if $B$ is known. Due to concavity, it satisfies $S_{f}(A \mid B) \leq S_{f}(A)=\sum_{i} f\left(q_{i}\right)$ (with $\left.q_{i}=p(A=i)=\sum_{j} p_{i j}\right) \forall S_{f}$. The difference

$$
I_{f}(A \mid B)=S_{f}(A)-S_{f}(A \mid B),
$$

is then non-negative, vanishing only if $p_{i j} / p_{j}=p_{i} \forall i, j$ with $p_{j}>0$, i.e., only if $A$ and $B$ are independent. It represents the uncertainty reduction (or generalized "information gain") about $A$ generated by the knowledge of $B$.

In the Shannon case $f(p)=-p \log _{a} p$, A3 becomes $S(A \mid B)=S(A, B)-S(B)$, where $S(A, B)=$ $-\sum_{i, j} p_{i j} \log _{a} p_{i j}, S(B)=-\sum_{j} p_{j} \log _{a} p_{j}$, but such relation no longer holds for a general $S_{f}$. Hence, while in the (classical) Shannon case $I(A \mid B)=S(A)+S(B)-$ $S(A, B)=I(B \mid A)$ is the mutual information, for a general $S_{f}, I_{f}(A \mid B)$ will differ in general from $I_{f}(B \mid A)$. Generalizations of the Shannon conditional entropy based on the Renyi entropy were recently discussed in 55. (and quantum versions in 36, 37), whereas special extensions for the Tsallis case were considered in 38].

\section{Appendix B: Relation with the entanglement of formation}

Let us sketch the proof of the identity (17) 23 26. Starting from the $(A C, B)$ Schmidt decomposition of the pure global state,

$$
\left|\Psi_{A C B}\right\rangle=\sum_{k=1}^{n} \sqrt{q_{k}}\left|\tilde{k}_{A C}\right\rangle\left|\tilde{k}_{B}\right\rangle,
$$

the state of $A C$ after a measurement in $B$ based on the operators (11) with outcome $j$ is the pure state (Eq. (13))

$$
\left|j_{A C}\right\rangle=\left(r_{j} / p_{j}\right)^{1 / 2} \sum_{k} \sqrt{q_{k}}\left\langle j_{B} \mid \tilde{k}_{B}\right\rangle\left|\tilde{k}_{A C}\right\rangle .
$$

Hence, $\rho_{A / \Pi_{j}}$ is the reduced state $\rho_{A}^{j}$ of $A$ in $\left|j_{A C}\right\rangle$ and $S_{f}\left(A \mid B_{\left\{\Pi_{j}\right\}}\right)=\sum_{j} p_{j} S_{f}\left(\rho_{A}^{j}\right)$ coincides then with the average entanglement of the decomposition $\rho_{A C}=$ $\sum_{j} p_{j} \rho_{A C / \Pi_{j}}$, where $\rho_{A C / \Pi_{j}}=\left|j_{A C}\right\rangle\left\langle j_{A C}\right|$. Conversely, Eq. (B1) implies that the states $\left|j_{A C}\right\rangle$ in any decomposition $\rho_{A C}=\sum_{j} p_{j}\left|j_{A C}\right\rangle\left\langle j_{A C}\right|$ (with $p_{j}>0$ ) should satisfy

$$
\sqrt{p_{j}}\left|j_{A C}\right\rangle=\sum_{k} U_{j k} \sqrt{q_{k}}\left|\tilde{k}_{A C}\right\rangle,
$$

where $U$ is an $m \times n$ matrix with orthonormal columns $\left(\sum_{j} U_{j k}^{*} U_{j k^{\prime}}=\delta_{k k^{\prime}}\right)$ and $m \geq n$. Comparison with Eq. (B2) indicates that we may identify such decomposition with that for a local measurement at $B$ with the operators (11), provided

$$
\sqrt{r_{j}}\left|j_{B}\right\rangle=\sum_{k} U_{j k}^{*}\left|\tilde{k}_{B}\right\rangle
$$


such that $U_{j k}=\sqrt{r_{j}}\left\langle j_{B} \mid \tilde{k}_{B}\right\rangle$. The ensuing operators $\Pi_{j}^{B}=r_{j}\left|j_{B}\right\rangle\left\langle j_{B}\right|$ form a valid POVM since $\sum_{j} \Pi_{j}^{B}=$ $\sum_{j, k, k^{\prime}} U_{j k}^{*} U_{j k^{\prime}}\left|\tilde{k}_{B}\right\rangle\left\langle\tilde{k}_{B}^{\prime}\left|=\sum_{k}\right| \tilde{k}_{B}\right\rangle\left\langle\tilde{k}_{B}\right|=I_{B}$ (assuming $\left.n=d_{B}\right)$.
[1] Werner R F 1989 Phys. Rev. A 404277

[2] Ollivier H and Zurek W H 2001 Phys. Rev. Lett. 88 017901

[3] Henderson L, Vedral V 2001 J. Phys. A 34 6899; Vedral V 2003 Phys. Rev. Lett. 90050401

[4] Zurek W H (2003) Phys. Rev. A 67 012320; 2003 Rev. Mod. Phys. 75715

[5] Knill E and Laflamme R 1998 Phys. Rev. Lett. 815672

[6] Datta A, Flammia S T and Caves C M 2005 Phys. Rev. A $\mathbf{7 2} 042316$

[7] Datta A, Shaji A and Caves C M 2008 Phys. Rev. Lett. 100050502

[8] Modi K et al 2012 Rev. Mod. Phys. 841655

[9] Streltsov A, Kampermann H and Bruß D 2011 Phys. Rev. Lett. 106160401

[10] Piani M et al 2011 Phys. Rev. Lett. 106220403

[11] Girolami D, Tufarelli T, Adesso G 2013 Phys. Rev. Lett. 110 240402; Adesso G et al, arXiv:1308.1680

[12] Wehrl A 1978 Rev. Mod. Phys. 50221

[13] Horodecki M et al 2005 Phys. Rev. A 71 062307; Oppenheim J et al 2002 Phys. Rev. Lett. 89180402

[14] Luo S 2008 Phys. Rev. A 77042303

[15] Modi K et al 2010 Phys. Rev. Lett. 104080501

[16] Dakić B, Vedral V and Brukner Č 2010 Phys. Rev. Lett. 105190502

[17] Rossignoli R, Canosa N, Ciliberti L 2010 Phys. Rev. A 82 052342; 2011 Phys. Rev. A 84052329

[18] Bellomo B et al 2012 Phys. Rev. A 85032104

[19] Girolami D and Adesso G 2012 Phys. Rev. Lett. 108 150403

[20] Luo S, Fu S, Oh C H 2012 Phys. Rev. A 85 032117; Chang L, Luo S 2013 Phys. Rev. A 87062303

[21] Paula F M, de Oliveira T R, Sarandy M S 2013 Phys. Rev. A 87064101

[22] Adesso G, Girolami D, Serafini A 2012 Phys. Rev. Lett. 109190502

[23] Koashi M, Winter A 2004 Phys. Rev. A 69022309

[24] Cavalcanti D et al 2011 Phys. Rev. A 83032324

[25] Madhok V and Datta A 2011 Phys. Rev. A 83032323

[26] Fanchini F F et al 2011 Phys. Rev. A 84012313

[27] Giorda P, Paris MGA 2010 Phys. Rev. Lett. 105020503

[28] Adesso G, Datta A 2010 Phys. Rev. Lett. 105030501 ; Mista L et al 2011 Phys. Rev. A $\mathbf{8 3} 042325$

[29] Fanchini F et al 2010 Phys. Rev. A 81052107

[30] M. Ali, A.R.P. Rau, G. Alber, Phys. Rev. A 81, 042105 (2010); A 82, 069902 (E) (2010);
[31] Ciliberti L, Rossignoli R, Canosa N 2010 Phys. Rev. A 82042316

[32] Girolami D, Adesso G 2011 Phys. Rev. A 83052108

[33] Lu X M et al 2011 Phys. Rev. A 83 012327; Huang Y 2013 Phys. Rev. A $\mathbf{8 8} 014302$

[34] Campbell S et al 2013 New J. of Phys. 15043033

[35] Canosa N, Rossignoli R 2002 Phys. Rev. Lett. 88 170401; 1999 Phys. Lett. A 264148

[36] Rastegin A, arXiv:1309.6048

[37] Müller-Lennert M et al, arXiv: 1306.3142.

[38] Abe S, Rajagopal A K 2001 Phys. A 289 157; Rajagopal A K et al, arXiv: 1309.6944

[39] Filip R 2002 Phys. Rev. A 65 062320; Nakazato H et al 2012, Phys. Rev. A 85 042316; Tanaka T et al 2013 Phys. Rev. A 87012303

[40] Nielsen M A, Chuang I L 2000 Quantum Computation and Quantum Information, Cambridge University Press (UK)

[41] Rungta P, Caves C M 2003 Phys. Rev. A I 67 012307; 2001 Phys. Rev. A 64042315

[42] Vidal G 2000 J. Mod. Opt 47355

[43] Bennett C H, DiVincenzo D P, Smolin J A, Wootters W K 1996 Phys. Rev. A 543824

[44] Tsallis C 1988 J. Stat. Phys 52 479; Curado E M F, Tsallis C 1991 J. Phys. A L69; Tsallis C 2009 Introduction to non-extensive statistical mechanics, Springer

[45] Majtey A P et al 2012 Phys. A 391 2491; Jurkowski J 2013 Int. J. Quantum Inf. 111350013.

[46] Nielsen M A 1999 Phys. Rev. Lett. 83436

[47] Hill S, Wootters W K 1997 Phys. Rev. Lett. 78 5022; Wootters W K 1998 Phys. Rev. Lett. 802245

[48] Girolami D, Adesso G 2011 Phys. Rev. A 84052110

[49] Zyczkowski K et al 1998 Phys. Rev. A 58 883; Vidal G, Werner R F 2002 Phys. Rev. A 65032314

[50] Rossignoli R, Canosa N, Matera J M 2008 Phys. Rev. A 77052322

[51] Giampaolo S M, Adesso G, Illuminati F 2009 Phys. Rev. B 79 224434; 2008 Phys. Rev. Lett. 100197201

[52] Ciliberti L, Canosa N, Rossignoli R 2013 Phys. Rev. A 88 012119; Canosa N, Ciliberti L, Rossignoli R 2013 Int.J.Mod.Phys. B 271345033

[53] Bhatia R 1997 Matrix Analysis, Springer (NY)

[54] Rossignoli R and Canosa N 2003 Phys. Rev. A 67 042302; 2002 Phys. Rev. A 66042306.

[55] Texeira A, Matos A, Antunes L 2012 IEEE Trans. Inf. Theory $\mathbf{5 8} 4273$. 